\title{
Cuantificación de hormonas $17 \beta$ - estradiol y progesterona en el caracol rosa Lobatus gigas (Mollusca, Gastropoda)
}

\author{
Fabiola Chong Sánchez, Martha Enríquez Díaz \& Dalila Aldana Aranda \\ Centro de Investigación y de Estudios Avanzados - Unidad Mérida, Laboratorio de Biología y Cultivo de Moluscos, \\ Antigua Carretera a Progreso Km. 6, 97310 Mérida, Yucatán, México; fabiola.chong@gmail.com, \\ marthaenriquez_1999@yahoo.com, daldana@cinvestav.mx
}

Recibido 17-X-2018. Corregido 02-V-2019. Aceptado 13-VI-2019.

\begin{abstract}
Quantification of $17 \beta$ - estradiol and progesterone hormones in the pink snail Lobatus gigas (Mollusca, Gastropoda). Quantification of steroid hormones in molluses is performed with different techniques, using the hemolymph or gonads. Lobatus gigas is a Caribbean gastropod of commercial interest indexed in CITES as a protected species. Hormonal studies of this species are as yet unavailable. The objective of this study is to determine the presence of the steroid hormones 173 - estradiol and progesterone in L. gigas using a non-invasive method, and to compare two techniques for their quantification. Every two months over the course of one year, the feces of ten organisms were collected in Xel-Ha park Quintana Roo, México. The samples were analyzed with High resolution liquid chromatography and Enzyme-linked immunosorbent assays. The values of both hormones were highest during the months of March to September then decreased during November and January. Comparison of the concentrations obtained with HPLC and EIA, presented similar results for $17 \beta$-estradiol (Passing - Bablock $\mathrm{r}=0.673$; mean differences $-0.17 \mathrm{ng} / \mathrm{ml}$ ). In contrast, the progesterone results with both techniques showed no adjustment (Passing - Bablock $\mathrm{r}=0.389$; mean differences $-1.43 \mathrm{ng} / \mathrm{ml}$ ). Our results suggest that the enzyme-linked immunosorbent assay is suitable for the study of hormones in L. gigas. The knowledge generated will allow the monitoring and selection of breeding organisms that are conditioned in laboratories and thus will not affect the collection of wild egg masses.
\end{abstract}

Key words: sexual hormones; Lobatus; aquaculture; reproduction; 17ß - estradiol; progesterone.

Chong Sánchez, F., Enríquez Díaz, M., \& Dalila Aldana Aranda, D. (2019). Cuantificación de hormonas $17 \beta$ - estradiol y progesterona en el caracol rosa Lobatus gigas (Mollusca, Gastropoda). Revista de Biología Tropical, 67(4), 708-715.

Las hormonas esteroideas sexuales $17 ß$ - estradiol y progesterona actúan como mensajeros químicos y participan en el control de procesos como la diferenciación sexual, el mantenimiento de las características sexuales, la maduración de gametos y el comportamiento sexual (Norman \& Henry, 2015; Ogino, Sato, \& Iguchi, 2016; Vardanyan \& Hruby, 2016). La presencia y biosíntesis de estas hormonas se ha demostrado en diferentes invertebrados (Janer, 2006; Fernandes, Loi, \& Porte, 2011).
En moluscos marinos la biosíntesis de $17 \AA$ - estradiol está registrada en Haliotis diversicolor (Zhou et al., 2011), Helix aspersa (Le Guellec et al., 1987) y en Clamys farreri (Liu, Xu, Robinson, Qin, \& Li, 2014); así como la biosíntesis de progesterona en Clione antartica (Hines, Bryan, Wasson, McClintock, \& Watts, 1996). Sin embargo, las funciones que desempeñan estas hormonas en los moluscos aún no han quedado del todo claras, aunque su presencia se relaciona con los eventos reproductivos. 
Las concentraciones más altas de $17 ß$ - estradiol se han encontrado en los periodos de madurez de hembras de Crassostrea gigas (Matzumoto, Osada, Osawa, \& Mori, 1997) y Sinonovacula constricta (Yan, Liu, Ke, Yu, \& Kong, 2011). Por otra parte las concentraciones de progesterona incrementan durante la etapa de gametogénesis en Biomphalaria alexandrina (Omran, 2008).

En acuacultura el uso de hormonas ha permitido mejorar la calidad de los gametos, producción y control de la madurez gonádica de cultivos de reproductores (Mylonas, Duncan, \& Asturiano, 2017; Wang et al., 2008). En el bivalvo Crassostrea gigas al inyectar $17 ß$ - estradiol en la gónada se incrementa el diámetro de los ovocitos y la producción de vitelo (Li, Osada, Suzuki, \& Mori, 1998) y en el caso de Placopecten magellanicus sirve para inducir el desove en (Wang \& Croll, 2006). Hasta el momento, no hay registros sobre la implementación de estas hormonas en gasterópodos con fines reproductivos.

El caracol rosa (L. gigas) es una especie del Caribe que representa un negocio lucrativo para los mercados de Estados Unidos y Europa (Acosta, 2006; FAO, 2010). No obstante, su sobreexplotación ha provocado el declive de sus poblaciones, por lo que desde 1992 se encuentra en la lista de animales protegidos (Apéndice II Cites). El desarrollo de técnicas para su cultivo de la etapa larval hasta juveniles, comenzó desde mediados de los años 70 y continuó hasta principios de los años 90 (Davis, 2000). Desde ese momento la larvicultura tuvo un constante desarrollo, dejando a un lado a los reproductores. Si bien Shawl y Davis (2004) describen la obtención de huevos al colocar hembras y machos de esta especie en un área confinada, no se han sido realizados más estudios sobre el establecimiento del cultivo de reproductores e inducción al desove.

A comparación del amplio desarrollo de técnicas para la mejora reproductiva en otras especies de moluscos con casos similares e.g. Haliotis spp. y Trochus niloticus, la información en el caracol rosa es limitada. La única granja de cultivo activa de L. gigas depende de la temporalidad reproductiva y la colecta de masas de huevos del medio natural para continuar con el ciclo. A pesar de que el control de la reproducción es un elemento necesario para un desarrollo sostenible de esta actividad (Mylonas et al., 2017). Por ello el objetivo del presente trabajo es determinar la presencia de las hormonas esteroides $17 ß$ - estradiol y progesterona en L. gigas a través de un método no invasivo y comparar dos técnicas para su cuantificación. Con base en los aportes de este estudio se determinará la viabilidad de ambas técnicas, costos, requerimientos, facilidad de manejo, y tiempo de análisis con el fin de continuar con los estudios reproductivos de esta especie.

\section{MATERIALES Y MÉTODOS}

Área y recolecta de muestras: Organismos adultos de L. gigas $(\mathrm{n}=10)$ fueron recolectados a través de buceo libre cada dos meses durante un año en la caleta del parque Xel- Há, Quintana Roo, México (20¹8'50"-

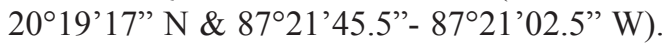
Posteriormente se determinó el sexo y se colocaron en acuarios por un lapso de 5 horas, a una densidad de un organismo en $40 \mathrm{~L}$ de agua de mar. Las heces emitidas se recolectaron de forma individual con una pipeta Pasteur y se conservaron a una temperatura de entre 2 y $4^{\circ}$ C para su traslado al laboratorio de Biología y Cultivo de Moluscos del Cinvestav Mérida. Al finalizar la toma de muestras los organismos fueron regresados al sitio de colecta.

Extracción de hormonas esteroideas: Un gramo de heces fue pesado y mezclado con $10 \mathrm{ml}$ de metanol al $80 \%$ (Palme, Touma, Arias, Dominchin, \& Lepschy, 2013). Esta mezcla fue homogenizada por de agitación manual; posterior a su sedimentación, el sobrenadante fue recuperado, filtrado (Millex-GV $0.22 \mu \mathrm{m}$ ) y dividido en dos frascos (uno para cada técnica). Las muestras se preservaron a $-20{ }^{\circ} \mathrm{C}$ hasta su análisis. 
Enzimoinmunoensayo (EIA): Las concentraciones de $17 \beta$ - estradiol y progesterona en heces de $L$. gigas fueron medidas usando el kit de EIA multi especie DetecX (Arbor Assay Inc.) específico para cada hormona. Este Kit permite medir la concentración a través de la unión de la hormona a un anticuerpo y un marcador (tetrametilbenzidina) con el cual se obtiene una reacción colorimétrica medible a través de espectrofotometría. La reactividad cruzada con metabolitos de cada hormona reportada es máxima de $0.02 \%$.

Para llevar a cabo la cuantificación de hormonas se estableció una curva de concentraciones estándar a partir diluciones de una solución patrón. De cada muestra se tomaron $100 \mu \mathrm{l}$ y se diluyeron en $400 \mu \mathrm{l}$ de Buffer de ensayo, de esta mezcla $50 \mu \mathrm{l}$ se analizaron por duplicado siguiendo las instrucciones del fabricante. La reacción colorimétrica se midió a $450 \mathrm{~nm}$ en un espectrofotómetro de placas (Dynex Technologies). Las densidades ópticas registradas se analizaron con el software MyAssays (MyAssays Ltd), el cual calcula la concentración a través de una regresión logística de cuatro parámetros a partir de la curva de calibración. El resultado obtenido se multiplicó por el factor de dilución, el cual se calculó dividiendo el volumen final entre el volumen inicial de la muestra (Helms, 2008).

Cromatografía liquida de alta eficacia (HPLC): Los reactivos químicos como 173 estradiol y progesterona ( $99 \%$ de pureza), agua y metanol grado HPLC (99.8 \% de pureza) se adquirieron en Sigma (St. Louis, MO, USA). El equipo constaba de una bomba de pistón reciproca de doble acción (LC-20AT; Shimadzu, Japan), un automuestreador (SIL-20A; Shimadzu, Japan) con control de temperatura (CCTO-20A; Shimadzu, Japan), un detector de luz ultravioleta (SpD-20A; Shimadzu, Japan) y una columna C18 $(5 \mu \mathrm{m}, 150$ x $46 \mathrm{~mm}$, Restek, Bellenfonte, PA, USA). Para llevar a cabo el análisis de las muestras, la columna y el sistema de HPLC se mantuvieron a $22{ }^{\circ} \mathrm{C}$ con un volumen prefijado de $50 \mu \mathrm{l}$ de inyección de la muestra. Se identificó el tiempo de retención y la tasa de flujo para cada hormona inyectando una solución patrón de $17 ß$ - estradiol y una de progesterona. Posteriormente se construyeron las curvas de concentración estándar a partir de diluciones de las soluciones patrón. Las muestras para la detección de $17 ß$ - estradiol se inyectaron por duplicado a un flujo de 0.8 $\mathrm{ml} / \mathrm{min}$, con una fase móvil de metanol / agua, a razón de $50 / 50 \mathrm{v} / \mathrm{v}$ y el detector UV fijado a $230 \mathrm{~nm}$. En el caso de progesterona se utilizó una fase móvil de 70 metanol / 30 agua $\mathrm{v} / \mathrm{v}$ y se inyectaron las muestras por duplicado a un flujo de $1 \mathrm{ml} / \mathrm{min}$ y con el detector UV fijado a $254 \mathrm{~nm}$. Entre cada muestra se analizaron blancos de metanol como vehículo para detectar si había interferencias en la elución. Los cromatogramas de la curva de calibración y de las muestras fueron analizados a través del software Shimadzu LC Solutions para calcular la concentración de cada muestra.

Métodos estadísticos: Previo a su análisis la normalidad de los datos fue comprobada con la prueba de Shapiro - Wilks. La comparación de ambas técnicas se llevó a cabo usando el método de ajuste de Passing - Bablok (Passing \& Bablok, 1983) junto con el gráfico de diferencias de Bland - Altman (Bland \& Altman, 1986) y una prueba t- Student (Sokal, 1995) para la comparabilidad entre técnicas. La comparación de las concentraciones para cada hormona en el tiempo se llevó a cabo con análisis de varianza (ANOVA) y comparación de Tukey (Sokal, 1995). Estas pruebas estadísticas se llevaron a cabo con el software Analyse it (Analyse it Software, Lt).

\section{RESULTADOS}

$17 ß$ - estradiol EIA versus HPLC: Las concentraciones de esta hormona presentaron una variación temporal (Fig. 1A). De marzo a julio se mantuvieron constantes, con concentraciones entre 0.3 a $3.6 \mathrm{ng} / \mathrm{ml}$ con EIA y 0.4 a $2.1 \mathrm{ng} / \mathrm{ml}$ con HPLC. De septiembre a enero las concentraciones disminuyeron significativamente $(\mathrm{P}<0.0001)$. 

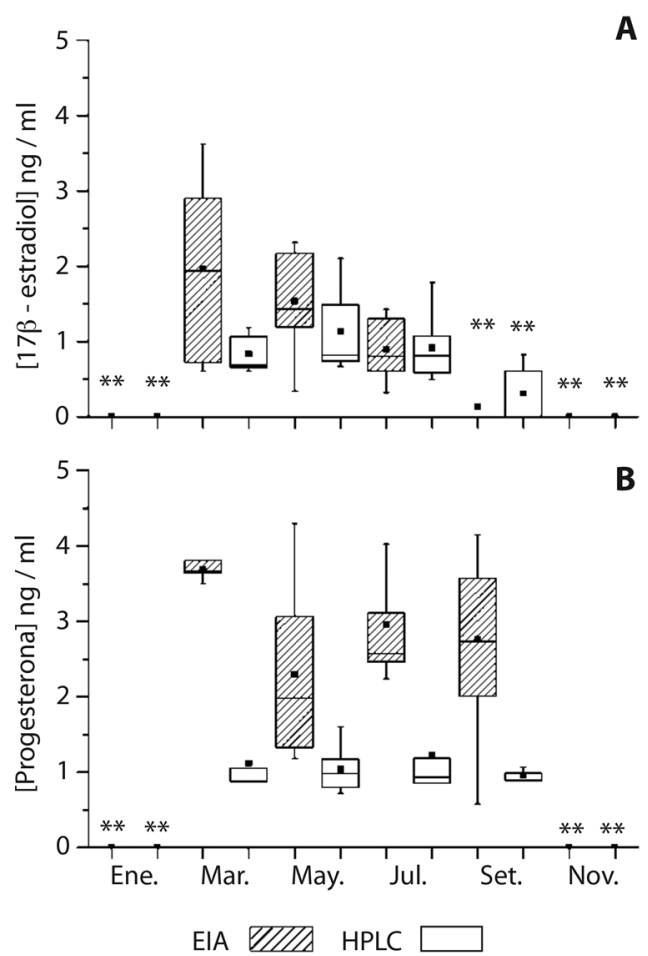

Fig. 1. Gráficas de cajas de la concentración de las hormonas 17ß - estradiol (A) y progesterona (B) en heces de Lobatus gigas en un periodo de un año, usando dos técnicas: Enzimoinmunoensayo (EIA) y Cromatografía líquida de alta resolución (HPLC). (**) representa las diferencias significativas entre meses para cada técnica $(\mathrm{P}$ $<0.0001)$.

La técnica de EIA se tomó como la técnica de referencia. Con el método de Passing - Bablock se obtuvo la ecuación con una pendiente $\mathrm{b}=0.5805$ (IC $95 \% 0.3785$ a 1.083 ) y una correlación $r=0.673$ (Fig. 2A). El ajuste entre ambas técnicas sugiere que existe diferencia significativa en la pendiente $(\mathrm{t}=-8.58, \mathrm{P}$ $<0.0001)$. El análisis por diferencia de medias de Bland - Altman mostro que la concentración media de $17 ß$ - estradiol con HPLC es de -0.17 ng / ml en comparación a la media de la concentración obtenida con EIA, y son comprables $(\mathrm{t}=-1.76, \mathrm{P}=0.08)$ (Fig. 2B).

Progesterona EIA versus HPLC: La variación temporal de esta hormona presento concentraciones mínimas y máximas de 0.5 a $4.30 \mathrm{ng} / \mathrm{ml}$ con EIA y de 0.7 a $1.9 \mathrm{ng} / \mathrm{ml}$ con HPLC de marzo a septiembre, las concentraciones durante los meses de enero y noviembre disminuyeron significativamente $(\mathrm{P}<0.0001)$ (Fig. 1B).

$\mathrm{Al}$ igual que el caso anterior la técnica de EIA para progesterona se tomó como técnica de referencia. Al analizar las concentraciones obtenidas con el método de Passing - Bablock se obtuvo una pendiente $\mathrm{b}=0.1849$ (IC $95 \%$ -1.832318 a -1.043762) y una correlación $\mathrm{r}=$ 0.389 (Fig. 2C). La comparación de métodos fue significativamente diferente en la pendiente $(\mathrm{t}=-20 ; \mathrm{P}<0.0001)$. El análisis de Bland - Altman indico que la diferencia media de las concentraciones de progesterona de EIA respecto a las obtenidas con HPLC fue de $-1.43 \mathrm{ng} / \mathrm{ml}$ (Fig. 2D), con una diferencia significativa entre ambos métodos $(\mathrm{t}=-7.3 ; \mathrm{P}<0.0001)$.

\section{DISCUSIÓN}

El análisis hormonal en mamíferos terrestres y acuáticos se realiza por lo general en sangre y tejidos ( Budzinski, Devier, Labadie, \& Togola, 2006; Amaral, 2010). En invertebrados, como crustáceos y moluscos se utilizan la hemolinfa y las gónadas (Bose, Majumdar, \& Bhattacharya, 1997; Gorbushin \& Iakovleva, 2006; Huang, Ye, Han, \& Wang, 2009). En el caso de moluscos, la hemolinfa se extrae por punción cardíaca causando daños y la obtención de las gónadas implica el sacrificio del organismo (Matzumoto et al., 1997; Gorbushin \& Iakovleva, 2006).

En especies protegidas e indexadas en CITES como es el caso de L. gigas, su colecta y más aún su sacrificio, requieren la obtención de permisos. Por lo anterior, una alternativa para el estudio de hormonas son los métodos no invasivos, a través de las excretas (Busso \& Ruiz, 2011; Sheriff, Dantzer, Delehanty, Palme, \& Boonstra, 2011).

En moluscos, las hormonas esteroideas han sido cuantificadas con diferentes técnicas. Con HPLC se midió $17 ß$ - estradiol y estriol en las gónadas de hembras del ostión de 

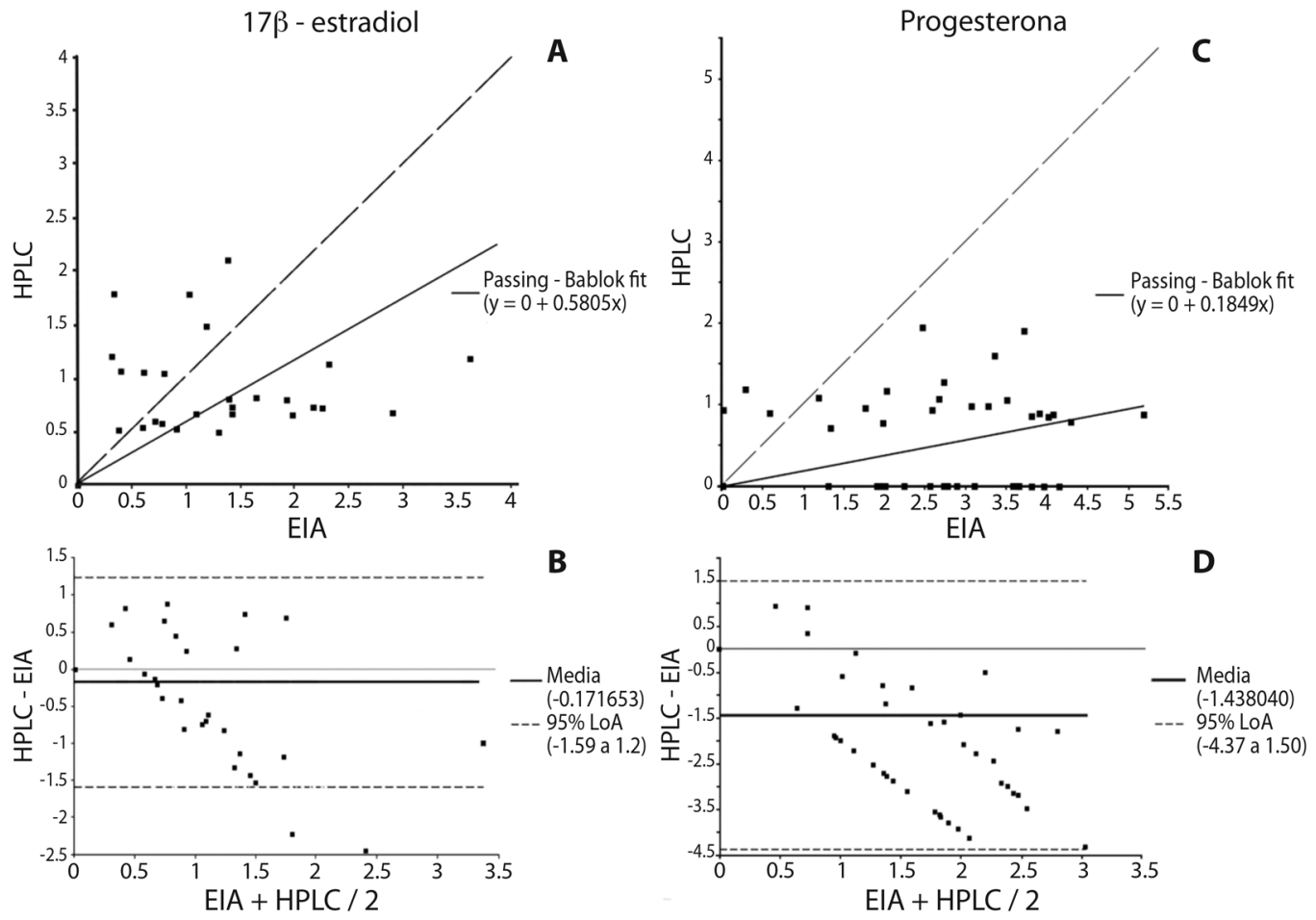

Fig. 2. Ajuste de Passing-Bablock y diferencia de medias de Bland - Altman de la concentración de las hormonas $17 ß$ estradiol (A, B) y progesterona (C, D) en Lobatus gigas con las técnicas de Enzimoinmunoensayo (EIA) y Cromatografía líquida de alta resolución (HPLC). Simbología (A, C): Misma escala (- -).

Crassostrea gigas durante la madurez sexual (Matzumoto et al., 1997). A través de HPLC y EIA se registraron las concentraciones más altas de progesterona, $17 ß$ - estradiol y testosterona durante el desove del bivalvo Mya arenaria (Siah, Pellerin, Benosman, Gagné, \& Amiard, 2002; Gauthier-Clerc, Pellerin, \& Amiard, 2006). En Achatina fulica con radioinmuno-ensayos se observó el incremento de17ß - estradiol en la madurez sexual (Bose et al., 1997).

En este estudio, con HPLC y EIA se cuantificaron las hormonas $17 ß$ - estradiol y progesterona. Las concentraciones de ambas variaron en el tiempo, siendo los meses de actividad reproductiva (marzo a septiembre) los que presentaron las concentraciones más altas, en comparación a los meses de reposo sexual (noviembre, febrero), cuyos valores fueron cercanos a cero. Estos resultados demuestran la presencia de un ciclo hormonal en L. gigas y su potencial influencia en el ciclo reproductivo de la especie.

Los resultados obtenidos en esta investigación para la progesterona con ambas técnicas fueron diferentes. Esto podría explicarse por la baja selectividad de HPLC para detectar esta hormona en las muestras (Cross \& Hornshaw, 2016).

Por lo que se refiere a la inversión para ambas técnicas, el HPLC tiene un costo entre 10000 a 25000 USD y la técnica de EIA de1 500 a 5000 USD. De esta última se adquieren paquetes que incluyen la hormona específica, los reactivos y los anticuerpos para realizar la reacción siendo de fácilmente el análisis. En cambio, HPLC requiere personal capacitado para calibración del cromatógrafo (Darwish, 2006; Cross \& Hornshaw, 2016).

Con base en lo anterior la técnica de EIA y el método no invasivo utilizado en este trabajo son una opción adecuada para el estudio 
de hormonas en moluscos, en particular de especies amenazadas como L. gigas. El conocimiento generado en este trabajo permitirá monitorear y seleccionar organismos reproductores que se encuentren acondicionados en laboratorios y de esta manera no incidir en la colecta de masas ovígeras silvestre (Li et al., 1998; Klaoudatos \& Klaoudatos, 2004; Mylonas et al., 2017).

\section{AGRADECIMIENTOS}

Los autores agradecen el apoyo a Eric Murillo Rodríguez del Laboratorio de Neurociencias Moleculares e Integrativas de la Universidad Anáhuac del Mayab por facilitar el equipo para llevar a cabo el análisis por HPLC y a Rossana Rodríguez Canul encargada del laboratorio de Inmunología y Biología Molecular del Cinvestav - Mérida por permitir el uso de sus instalaciones para llevar a cabo el análisis por EIA. Este trabajo fue apoyado por la beca otorgada por Conacyt a Fabiola Chong Sánchez.

\section{RESUMEN}

La cuantificación de las hormonas esteroides en moluscos se lleva a cabo con diferentes técnicas utilizando la hemolinfa o gónadas. Lobatus gigas es un gasterópodo de interés comercial en el Caribe, indexado en CITES como una especie protegida. Los estudios hormonales de esta especie aún no están disponibles. Por lo tanto, el objetivo de este estudio fue determinar la presencia de las hormonas esteroides $17 ß$ - estradiol y progesterona en L. gigas a través de un método no invasivo y comparar dos técnicas para su cuantificación. Cada dos meses en un período de un año, se recolectaron las heces de diez organismos en XelHá, Quintana Roo, México. Las muestras se analizaron con cromatografía líquida de alta resolución y EIA. Los valores más altos de ambas hormonas se presentaron de marzo a septiembre y disminuyeron en noviembre y enero. La comparación de las concentraciones obtenidas con HPLC y EIA, mostró que los resultados son similares para el $17 \beta$-estradiol (Passing - Bablock $\mathrm{r}=0.673 ;-0.17 \mathrm{ng} / \mathrm{ml}$ ). En contraste, los resultados de la progesterona con ambas técnicas no mostraron ajuste (Passing - Bablock $r=0.389$; $-1.43 \mathrm{ng} / \mathrm{ml}$ ). Nuestros resultados sugieren que la técnica de EIA es adecuada para el estudio de hormonas en esta especie. El conocimiento generado permitirá monitorear y seleccionar organismos reproductores que se encuentren acondicionados en laboratorios y de esta manera no incidir en la recolecta de masas ovígeras silvestres.

Palabras clave: hormonas sexuales; Lobatus; acuacultura; reproducción; $17 ß$ - estradiol; progesterona.

\section{REFERENCIAS}

Acosta, C. A. (2006). Impending trade suspensions of Caribbean Queen Conch under CITES. Fisheries, 31(12), 601-606. DOI: 10.1577/1548-8446(2006)31[601:ITSOCQ]2.0.CO;2

Amaral, R. S. (2010). Use of alternative matrices to monitor steroid hormones in aquatic mammals: A Review. Aquatic Mammals, 36(2), 162-171.

Bland, M. J., \& Altman, D. G. (1986). Statistical methods for assessing agreement between two methods of clinical measurement. The Lancet, 327(8476), 307-310. DOI: $10.1016 / \mathrm{S} 0140-6736(86) 90837-8$

Bose, R., Majumdar, C., \& Bhattacharya, S. (1997). Steroids in Achatina fulica (Bowdich): steroid profile in haemolymph and in vitro release of steroids from endogenous precursors by ovotestis and albumen gland. Comparative Biochemistry and Physiology Part C: Pharmacology, Toxicology and Endocrinology, 116(3), 179-182. DOI: 10.1016/ S0742-8413(96)00163-6

Budzinski, H., Devier, M. H., Labadie, P., \& Togola, A. (2006). Analysis of hormonal steroids in fish plasma and bile by coupling solid-phase extraction to GC/ MS. Analytical and Bioanalytical Chemistry, 386(5), 1429-1439. DOI: 10.1007/s00216-006-0686-9

Busso, J. M., \& Ruiz, R. D. (2011). Excretion of steroid hormones in rodents: An overview on species differences for new biomedical animal research models. In E. Diamanti-Kandarakis (Ed.), Contemporary Aspects of Endocrinology (pp. 375-396). DOI: 10.5772/17051

Cross, T. G., \& Hornshaw, M. P. (2016). Can LC and LC-MS ever replace immunoassays? Journal of Applied Bioanalysis, 2(4), 108-116. DOI: 10.17145/ jab.16.015

Darwish, I. A. (2006). Immunoassay methods and their applications in pharmaceutical Analysis: basic methodology and recent advances. International Journal of Biomedical Science: IJBS, 2(3), 217-235.

Davis, M. (2000). Queen conch (Strombus gigas) culture techniques for research, stock enhancement and grow out markets. In M. Fingerman \& R. Nagabhushanam (Eds.), Recent Advances in Marine Biotecnology, Seaweeds and Invertebrated (pp. 127-159). Enfield, N. H., USA: Science Publishers.

FAO. (2010). The state of world fisheries and aquaculture. Rome, Italy: FAO. 
Fernandes, D., Loi, B., \& Porte, C. (2011). Biosynthesis and metabolism of steroids in molluscs. The Journal of Steroid Biochemistry and Molecular Biology, 127(35), 189-195. DOI: 10.1016/j.jsbmb.2010.12.009

Gauthier-Clerc, S., Pellerin, J., \& Amiard, J. C. (2006). Estradiol- $17 \beta$ and testosterone concentrations in male and female Mya arenaria (Mollusca bivalvia) during the reproductive cycle. General and Comparative Endocrinology, 145(2), 133-139. DOI: 10.1016/j. ygcen.2005.08.004

Gorbushin, A. M., \& Iakovleva, N. V. (2006). Haemogram of Littorina littorea. Journal of the Marine Biological Association of the United Kingdom, 86(5), 11751181. DOI: $10.1017 / \mathrm{S} 0025315406014160$

Helms, J. R. (2008). Mathematics for Medical and clinical laboratory professionals. Boston, USA: Cengage Learning.

Hines, G. A., Bryan, P. J., Wasson, K. M., McClintock, J. B., \& Watts, S. A. (1996). Sex steroid metabolism in the Antarctic pteropod Clione antarctica (Mollusca: Gastropoda). Invertebrate Biology, 115(2), 113-119. DOI: $10.2307 / 3227042$

Huang, H., Ye, H., Han, S., \& Wang, G. (2009). Profiles of gonadotropins and steroid hormone-like substances in the hemolymph of mud crab Scylla paramamosain during the reproduction cycle. Marine and Freshwater Behaviour and Physiology, 42(4), 297-305. DOI: 10.1080/10236240903174792

Janer, G. (2006). Steroid levels, steroid metabolic pathways and their modulation by endocrine disruptors in invertebrates. Barcelona, España: Universidad Autónoma de Barcelona. Recuperado de https://digital. csic.es/handle/10261/23795

Klaoudatos, S., \& Klaoudatos, D. (2004). Brood stock formation of the hermaphrodite finfish species Pagellus erythrinus (common Pandora) from fish reared in captivity. Mediterranean Marine Science, 5(1), 187198. DOI: $10.12681 / \mathrm{mms} .224$

Le Guellec, D., Thiard, M. C., Remy-Martin, J. P., Deray, A., Gomot, L., \& Adessi, G. L. (1987). In vitro metabolism of androstenedione and identification of endogenous steroids in Helix aspersa. General and Comparative Endocrinology, 66(3), 425-433. DOI: 10.1016/0016-6480(87)90253-X

Li, Q., Osada, M., Suzuki, T., \& Mori, K. (1998). Changes in vitellin during oogenesis and effect of estradiol-17 $\beta$ on vitellogenesis in the Pacific oyster Crassostrea gigas. Invertebrate Reproduction \& Development, 33(1), 87-93. DOI: 10.1080/07924259.1998.9652345

Liu, Y., Xu, T., Robinson, N., Qin, J., \& Li, X. (2014). Cryopreservation of sperm in farmed Australian greenlip abalone Haliotis laevigata. Cryobiology, 68(2), 185-193. DOI: 10.1016/j.cryobiol.2014.01.002
Matzumoto, T., Osada, M., Osawa, Y., \& Mori, K. (1997). Gonadal estrogen profile and immunohistochemical localization of steroidogenic enzymes in the Oyster and Scallop during sexual maturation. Comparative Biochemistry and Physiology, 118(4), 811-817.

Mylonas, C. C., Duncan, N. J., \& Asturiano, J. F. (2017). Hormonal manipulations for the enhancement of sperm production in cultured fish and evaluation of sperm quality. Aquaculture, 472, 21-44. DOI: 10.1016/j.aquaculture.2016.04.021

Norman, A. W., \& Henry, H. L. (Eds.). (2015). Chapter 1 - Hormones: An Introduction. In Hormones (Third Edition, pp. 1-25). San Diego, USA: Academic Press. Retrieved from http://www.sciencedirect.com/ science/article/pii/B978008091906500001X

Ogino, Y., Sato, T., \& Iguchi, T. (2016). Gonadal steroids. In Y. Takei, H. Ando, \& K. Tsutsui (Eds.), Handbook of Hormones (pp. 504-506). San Diego, USA: Academic Press. DOI: 10.1016/B978-0-12-801028-0.00094-5

Omran, N. E. S. E. S. (2008). Studies on sex and gonadotropic hormones accompanied with ovotestis maturation of Biomphalaria alexandrina snails (Pulmonata: Basommatophora). Reproductive Biology, $50,365-382$.

Palme, R., Touma, C., Arias, N., Dominchin, M. F., \& Lepschy, M. (2013). Steroid extraction: Get the best out of faecal samples. Wiener Tierärztliche Monatsschrift Special Issue, 100(9-10), 238-246.

Passing, B., \& Bablok, W. (1983). A new biometrical procedure for testing the equality of measurements from two different analytical methods application of linear regression procedures for method comparison studies in clinical chemistry. Journal of Clinical Chemistry and Clinical Biochemistry, 21, 709-720.

Shawl, M. D., \& Davis, M. (2004). Captive breeding behavior of four strombidae Conch. Journal of Shellfish Research, 23(1), 157-164.

Sheriff, M. J., Dantzer, B., Delehanty, B., Palme, R., \& Boonstra, R. (2011). Measuring stress in wildlife: techniques for quantifying glucocorticoids. Oecologia, 166(4), 869-887. DOI: 10.1007/ s00442-011-1943-y

Siah, A., Pellerin, J., Benosman, A., Gagné, J. P., \& Amiard, J. C. (2002). Seasonal gonad progesterone pattern in the soft-shell clam Mya arenaria. Comparative Biochemistry and Physiology Part A: Molecular \& Integrative Physiology, 132(2), 499-511. DOI: 10.1016/S1095-6433(02)00095-8

Sokal, R. R. \& Rohlf, F. J. (1995). Biometry: the principles and practice of statistics in Biological Research. $3 r d$ Edition. New York, USA: W.H. Freeman and Co.

Vardanyan, R., \& Hruby, V. (Eds.). (2016). Steroid hormones. In Synthesis of best-seller drugs (pp. 459-493). 
Boston, USA: Academic Press. DOI: 10.1016/ B978-0-12-411492-0.00027-4

Wang, C., \& Croll, R. P. (2006). Effects of sex steroids on spawning in the sea scallop, Placopecten magellanicus. Aquaculture, 256(1-4), 423-432. DOI: 10.1016/j. aquaculture.2006.01.017

Wang, H. P., Gao, Z., Beres, B., Ottobre, J., Wallat, G., Tiu, L., ... Yao, H. (2008). Effects of estradiol-17 $\beta$ on survival, growth performance, sex reversal and gonadal structure of bluegill sunfish Lepomis macrochirus. Aquaculture, 285(1), 216-223. DOI: 10.1016/j. aquaculture.2008.08.041
Yan, H., Liu, W., Ke, Q., Yu, R., \& Kong, L. (2011). Seasonal changes of oestradiol-17b and testosterone concentrations in the gonad of the Razor Clam Sinonovacula Constricta (Lamarck, 1818). Journal of Molluscan Studies, 77, 116-122. DOI: 10.1093/ mollus/eyq045

Zhou, J., Gao, Y. F., Li, L., Zhai, H. N., Tan, S. J., \& Cai, Z. H. (2011). Identification and functional characterization of a putative $17 \beta$-hydroxysteroid dehydrogenase 12 in abalone (Haliotis diversicolor). Molecular and Cellular Biochemistry, 354(1-2), 123-133. DOI: 10.1007/s11010-011-0811-8 